\title{
THE CYLINDRICAL CELL APPROXIMATION IN THERMAL UTILIZATION*
}

\author{
P. F. ZweIFel and M. A. Mannan $\dagger$ \\ The University of Michigan, Ann Arbor, Michigan
}

(Received 11 August 1966)

\begin{abstract}
In solving the neutron diffusion equation in one cell of an infinite lattice, a common procedure is to replace the rectangular (or hexagonal) cell with an equivalent cylindrical cell. In order to estimate the effect of this approximation a new method is presented in which the flux in the entire cell (not the equivalent cylindrical cell) is expanded in a Fourier cosine series. Then the boundary conditions over the cell surface are satisfied exactly. Furthermore, algebraic equations for the expansion coefficients of the flux are obtained because the integrals over the cell can be done in closed form. It is found that truncation of the flux series after 10 terms gives good results. The method is felt to have more general application than to the actual problem treated here since such 'mixed geometry" problems frequently arise in reactor physics and in other areas as well.
\end{abstract}

\section{INTRODUCTION}

IN THE calculation of such quantities as thermal utilization in rodded reactor lattices, it is customary to solve the diffusion equation in one 'cell' of the system, imposing a zero current boundary condition over the cell boundary (GLASSTONE and EDLUND, 1952). Because the fuel rods are frequently cylindrical, while the cell is generally rectangular or hexagonal, such a calculation is difficult to perform, and it is customary to replace the actual cell by an 'equivalent cylindrical cell'. A number of authors (WeingerG, 1942; Clark and NewmarCh, 1955; Galanin, 1956; NeumanN, 1957; PAZY and Goshen, 1961; AMOUYAL et al., 1957) have studied the error introduced by replacing the straight line segments of the actual cell by a circle, using several techniques which we shall not attempt to review here.

We report here a new analytic approach to this problem, which is, in some sense related to the work of Clark and Newmarch, although it is somewhat simpler. In effect, we assume diffusion theory to be valid both in fuel and moderator, and expand the flux in the entire cell in a Fourier series. In this way the zero-current boundary condition on the surface of the cell can be satisfied exactly. (Then, we do not need, as Neumann does, to consider the contribution of other cells.) Then, we are led to an infinite set of coupled equations to solve for the expansion coefficients of the flux, although we find that a reasonably small number of terms represents a good approximation.

In Section 2 we sketch the mathematical details of our work, and then, in Section 3 we give some numerical results. Finally, in Section 4 we comment briefly on some other possible applications of the method. In particular, we feel that the general approach described here for solving the diffusion equation in 'mixed' geometries (such as the cylindrical fuel rod in a rectangular cell) is of mathematical interest, quite apart from the practical application described in this paper. The fact that

* Based on a portion of a doctoral thesis submitted by one of the authors (M. A. M.) to The University of Michigan. A preliminary account of this work was reported at the Salt Lake City meeting of the American Nuclear Society. [(1963) Trans. Am. Nucl. Soc. 6, 4.]

$\dagger$ Present address: Pakistan Institute of Nuclear Science and Technology, Rawalpindi, Pakistan. 
analytical expressions can be found for the coefficients in the equations for the expansion coefficients of the flux is significant because it means that problems of this type can be solved by reduction to algebraic equations with tabulated coefficients. No numerical integrations and no reduction of differential equations to difference equations are required.

\section{MATHEMATICAL DETAILS}

We consider a rectangular cell of length $2 a$ along the $x$-axis and width $2 b$ along the $y$-axis with a fuel rod of radius $\rho$ at the centre (see Fig. 1). The diffusion equation for the neutron flux $\phi(x, y)$ can be written in the form

$$
-D(x, y)\left(\frac{\partial^{2} \phi}{\partial x^{2}}+\frac{\partial^{2} \phi}{\partial y^{2}}\right)-\frac{\partial D}{\partial x} \frac{\partial \phi}{\partial x}-\frac{\partial D}{\partial y} \frac{\partial \phi}{\partial y}+\Sigma_{a}(x, y) \phi(x, y)=S(x, y) .
$$

Here $D$ is the diffusion coefficient (assumed to take the constant value $D_{F}$ in the fuel and $D_{M}$ in the moderator), $\Sigma_{a}$ is the absorption cross section (with values $\Sigma_{a F}$ and $\left.\Sigma_{a M}\right)$ and $S(x, y)$ is a slowing down source assumed, as is customary (Glasstone and Edlund), to vanish in the fuel and to have a constant value $S$ in the moderator.

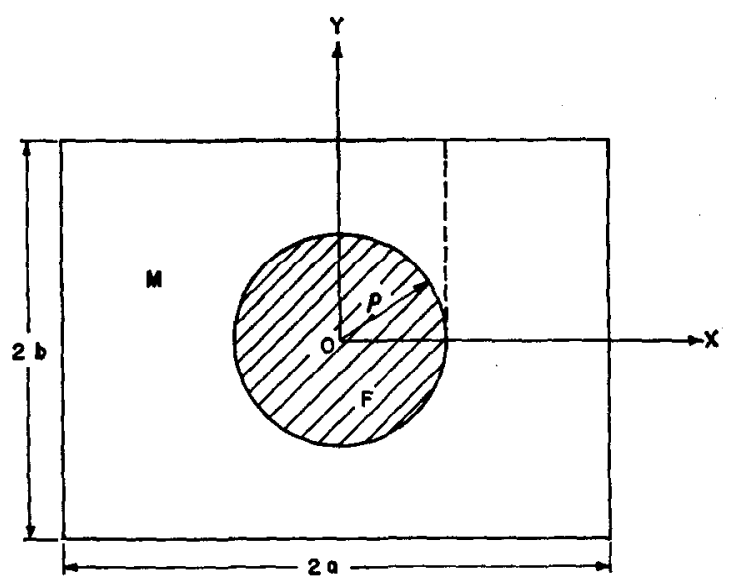

Fro. 1.-Cross section of rectangular cell.

We now expand the flux as

$$
\phi(x, y)=\frac{a_{00}}{4}+\sum_{k=0}^{\infty} \sum_{l=0}^{\infty} a_{k l} \cos \frac{k \pi x}{a} \cos \frac{l \pi y}{b} .
$$

In this way, the symmetry condition (about the cell centre) and the zero-current condition are automatically satisfied. The summation is over all values of $k$ and $l$ except the pair $k=l=0$.

The procedure now is to insert the expansion, equation (2), into (1), to multiply by

$$
\cos \frac{m \pi x}{a} \cos \frac{n \pi y}{b},
$$

and to integrate over the cell. The integrations over the terms involving $D(x, y)$, 
$\Sigma_{a}(x, y)$ and $S(x, y)$ are carried out by dividing the integration up into several parts, in each of which $D, S$ and $\Sigma_{a}$ are constant:

$$
\int_{0}^{a} \mathrm{~d} x \int_{0}^{b} \mathrm{~d} y=\int_{0}^{\rho} \mathrm{d} x \int_{0}^{\sqrt{\rho^{2}-x^{2}}} \mathrm{~d} y+\int_{0}^{\rho} \mathrm{d} x \int_{\sqrt{\rho^{2}-x^{2}}}^{b} \mathrm{~d} y+\int_{\rho}^{a} \mathrm{~d} x \int_{0}^{b} \mathrm{~d} y .
$$

In the first term on the right-hand side of equation (2), the integration is over the fuel region, while in the second and third term it is over the moderator region.

The integration involving the second and third terms on the left-hand side of equation (1) can be carried out by noting that $\partial D / \partial x$ and $\partial D / \partial y$ are proportional to delta functions:

$$
\frac{\partial D}{\partial x}=\left(D_{M}-D_{F}\right) \delta\left(x-\sqrt{\rho^{2}-y^{2}}\right)
$$

and

$$
\frac{\partial D}{\partial y}=\left(D_{M}-D_{F}\right) \delta\left(y-\sqrt{\rho^{2}-x^{2}}\right)
$$

After all of these integrations have been carried out, equation (1) becomes a set of coupled equations for the coefficients:

$$
\begin{array}{r}
a_{00}\left[\sum_{a M}\left(a b-\frac{\pi \rho^{2}}{4}\right) \delta_{0 m} \delta_{0 n}+\sum_{a F} \frac{\pi \rho^{2}}{4} \delta_{0 m} \delta_{0 n}-I\left(\sum_{a} ; 0,0, m, n\right)\right] \\
+\sum_{k=0}^{\infty} \sum_{l=0}^{\infty} a_{k l}\left[\left\{\left(\frac{k \pi}{a}\right)^{2}+\left(\frac{l \pi}{b}\right)^{2}\right\} I(D ; k, l, m, n)+\left(\frac{k \pi}{a}\right) H_{x}(k, l, m, n)\right. \\
\left.+\left(\frac{l \pi}{b}\right) H_{y}(k, l, m, n)+I\left(\sum_{a} ; k, l, m, n\right)\right]=S(m, n) .
\end{array}
$$

Here

$$
\begin{aligned}
I(f ; k, l, m, n) & =\iint_{\text {eell }} f(x, y)\left\{\cos \frac{k \pi x}{a} \cos \frac{l \pi y}{b} \cos \frac{m \pi x}{a} \cos \frac{n \pi y}{b}\right\} \mathrm{d} x \mathrm{~d} y, \\
H_{x}(k, l, m, n) & =\iint_{\text {cell }} \frac{\partial D}{\partial x}\left\{\sin \frac{k \pi x}{a} \cos \frac{l \pi y}{b} \cos \frac{m \pi x}{a} \cos \frac{n \pi y}{b}\right\} \mathrm{d} x \mathrm{~d} y \\
H_{y}(k, l, m, n) & =\iint_{\text {eell }} \frac{\partial D}{\partial y}\left\{\cos \frac{k \pi x}{a} \sin \frac{l \pi y}{b} \cos \frac{m \pi x}{a} \cos \frac{n \pi y}{b}\right\} \mathrm{d} x \mathrm{~d} y
\end{aligned}
$$

and

$$
\begin{aligned}
I(f ; 0,0, m, n) & =0, \quad m=n=0 \\
& =I(f ; 0,0, m, n) \quad \text { otherwise. }
\end{aligned}
$$

By breaking up the range of integrations, as displayed in equation (3), an explicit expression for $I(f ; k, l, m, n)$ can be obtained. The details are given elsewhere (MANNAN, 1964) although the appropriate formulae can also be found in standard 
mathematical texts (MORSE and FeSHBACH, 1953):

$$
\begin{aligned}
& I(f ; k, l, m, n)=\left(D_{F}-D_{M}\right)[F(k, l, m, n)+F(k, l, m,-n) \\
& \quad+F(k, l,-m, n)+F(k, l,-m,-n)]+a b \Lambda_{k l} \delta_{m k} \delta_{n l} .
\end{aligned}
$$

Here

$$
\begin{aligned}
& F(k, l, m, n)=J_{1}\left(\pi \rho \sqrt{\left(\frac{k+m}{a}\right)^{2}+\left(\frac{l+n}{b}\right)^{2}}\right) \\
& \times \frac{\rho}{2}\left[\left(\frac{k+m}{a}\right)^{2}+\left(\frac{l+n}{b}\right)^{2}\right]^{-1 / 2},
\end{aligned}
$$

where $J_{1}$ is the Bessel function of first order. Also

$$
\begin{aligned}
& \Lambda_{00}=4, \\
& \Lambda_{k 0}=\Lambda_{0 k}=2, \quad k \neq 0, \\
& \Lambda_{k l}=1, \quad k, l, \neq 0 .
\end{aligned}
$$

The functions $H_{x}$ and $H_{y}$ are also given in terms of the $F$ function:

$$
\begin{aligned}
H_{x}(k, l, m, n)=\left(D_{M}\right. & \left.-D_{F}\right)\left[\left(\frac{k+m}{a}\right) \pi\{F(k, l, m, n)+F(k, l, m,-n)\}\right. \\
& \left.+\left(\frac{k-m}{a}\right) \pi\{F(k, l,-m, n)+F(k, l,-m,-n)\}\right], \\
H_{y}(k, l, m, n)=\left(D_{M}-D_{F}\right)\left[\left(\frac{l+n}{b}\right) \pi\{F(k, l, m, n)+F(k, l,-m, n)\}\right. & \\
& \left.+\left(\frac{l-n}{b}\right) \pi\{F(k, l, m,-n)+F(k, l,-m,-n)\}\right] .
\end{aligned}
$$

Also

where

$$
S(m, n)=S\left[-4 F(0,0, m, n)+\left\{4 a b-\pi \rho^{2}\right\} \delta_{0 m} \delta_{0 n}\right]
$$

$$
\begin{aligned}
F(0,0, m, n) & =0, \quad m=n=0 \\
& =F(0,0, m, n) \quad \text { otherwise. }
\end{aligned}
$$

The procedure, then, is to solve the coupled set of equations (5) for the coefficients $a_{m n}$, and then to substitute these results into equation (2) to reconstruct the neutron flux in the cell.

\section{CAlCulations}

In solving equations (5), it is clearly necessary to truncate the set. In all of the calculations reported here the maximum values of the indices $k$ and $l$ were taken equal, ranging from 1 to 7 , in order to evaluate effect of truncation. (The cross scctions wcrc taken from ANL-5800). The solution of the systems of equations was carried out at the University of Michigan Computer facility on an IBM 7090 computer and the thermal utilization, $f$, was computed from the average fluxes in moderator fuel according to the usual formulas (Glasstone and Edlund). The equivalent cylindrical cell (ECC) was defined to have the same area as that of the rectangular cell, i.e. a radius $(4 a b / \pi)^{1 / 2}$. The results of the calculations are given in Tables $1-4$. 
TABLE 1.-THERMAL UTILIZATION OF A NATURAL URANIUM-GRAPHTtE CELL FOR VARIOUS FLUX APPROXIMATIONS $a=2 \mathrm{~cm}, b=2.5 \mathrm{~cm}, \rho=1 \mathrm{~cm}$

\begin{tabular}{|c|c|c|c|c|c|}
\hline$k$ & $l$ & $\begin{array}{c}\text { Average flux* } \\
\text { in the fuel } \\
\bar{\phi}_{F}\end{array}$ & $\begin{array}{c}\text { Average flux* } \\
\text { in the moderator } \\
\bar{\phi}_{M}\end{array}$ & $\bar{\phi}_{M} / \bar{\phi}_{F}$ & $\begin{array}{l}\text { Thermal utilization } \\
\qquad f\end{array}$ \\
\hline 1 & 1 & $14 \cdot 039043$ & $16 \cdot 524909$ & $1 \cdot 177068$ & 0.995510 \\
\hline 2 & 2 & $14 \cdot 060333$ & $16 \cdot 703461$ & $1 \cdot 187985$ & 0.995469 \\
\hline 3 & 3 & 14.075512 & $16 \cdot 736275$ & $1 \cdot 189035$ & 0.995465 \\
\hline 4 & 4 & 14.090440 & $16 \cdot 762192$ & $1 \cdot 189615$ & 0.995463 \\
\hline 5 & 5 & 14.099829 & $16 \cdot 774762$ & $1 \cdot 189714$ & 0.995462 \\
\hline 6 & 6 & $14 \cdot 105524$ & $16 \cdot 782078$ & $1 \cdot 189752$ & 0.995462 \\
\hline 7 & 7 & $14 \cdot 111894$ & $16 \cdot 789801$ & $1 \cdot 189762$ & 0.995462 \\
\hline
\end{tabular}

* Arbitrary units.

TABLE 2.-THERMAL UTILIZATION OF A NATURAL URANIUM-HEAVY WATER CELL FOR VARIOUS FLUX APPROXIMATIONS

$a=2 \mathrm{~cm}, b=2 \mathrm{~cm}, \rho=1 \mathrm{~cm}$

\begin{tabular}{|c|c|c|c|c|c|}
\hline$k$ & $l$ & $\begin{array}{c}\text { Average flux* } \\
\text { in the fuel } \\
\bar{\phi}_{F}\end{array}$ & $\begin{array}{c}\text { Average flux } \\
\text { in the moderator } \\
\bar{\phi}_{M}\end{array}$ & $\bar{\phi}_{M} / \bar{\phi}_{F}$ & $\begin{array}{l}\text { Thermal utilization } \\
f f\end{array}$ \\
\hline 1 & 1 & $9 \cdot 936120$ & $12 \cdot 057761$ & $1 \cdot 213528$ & 0.999550 \\
\hline 2 & 2 & $9 \cdot 893984$ & $12 \cdot 055554$ & $1 \cdot 218473$ & 0.999548 \\
\hline 3 & 3 & $9 \cdot 856714$ & 12.039424 & $1 \cdot 221444$ & 0.999547 \\
\hline 4 & 4 & $9 \cdot 849240$ & 12.034834 & $1 \cdot 221905$ & $0-999547$ \\
\hline 5 & 5 & $9 \cdot 840412$ & $12 \cdot 028025$ & $1 \cdot 222309$ & 0.999547 \\
\hline 6 & 6 & $9 \cdot 837569$ & $12 \cdot 025747$ & $1 \cdot 222431$ & 0.999547 \\
\hline 7 & 7 & $9 \cdot 833622$ & $12 \cdot 022149$ & $1 \cdot 222556$ & 0.999547 \\
\hline
\end{tabular}

* Arbitrary units.

TABLE 3.-COMPARISON OF A RECTANGULAR NATURAL URANIUM-GRAPHITE CELL WITH AN EQUIVALENT CYLINDRICAL CELL

$$
a=2 \mathrm{~cm}, b=2.5 \mathrm{~cm}, k_{\max }=l_{\max }=5
$$

\begin{tabular}{ccccc}
\hline$\rho(\mathrm{cm})$ & $\left(\frac{\bar{\phi}_{M}}{\bar{\phi}_{F}}\right)_{\mathrm{RC}}$ & $\left(\frac{\bar{\phi}_{M}}{\bar{\phi}_{P}}\right)_{\mathrm{ECC}}$ & $f_{\mathrm{RC}}$ & $f_{\mathbf{E C C}}$ \\
\hline 1.8 & 1.435236 & 1.300985 & 0.999012 & 0.998699 \\
1.6 & 1.353071 & 1.273241 & 0.998566 & 0.998568 \\
1.4 & 1.292296 & 1.242156 & 0.997930 & 0.998404 \\
1.2 & 1.239316 & 1.208218 & 0.996982 & 0.998189 \\
1.0 & 1.189714 & 1.171716 & 0.995462 & 0.997893 \\
0.8 & 1.142149 & 1.133084 & 0.992756 & 0.997454 \\
0.6 & 1.096617 & 1.093305 & 0.987104 & 0.996727 \\
0.4 & 1.054568 & 1.054131 & 0.971620 & 0.995273 \\
\hline
\end{tabular}

$\mathrm{RC}=$ Rectangular cell.

$\mathrm{ECC}=$ Equivalent cylindrical cell. 
TABle 4. - COMPARISON OF A RECTANGULAR NATURAL URANIUM-heAVY WATER CELL WTTH AN EQUTVALENT CYLINDRICAL CRLL $a=2 \mathrm{~cm}, b=2.5 \mathrm{~cm}, k_{\max }=l_{\max }=5$

\begin{tabular}{|c|c|c|c|c|}
\hline$\rho(\mathrm{cm})$ & $\left(\frac{\bar{\phi}_{\text {Mr }}}{\bar{\phi}_{F}}\right)_{\mathrm{RO}}$ & $\left(\frac{\bar{\phi}_{\mathbf{M}}}{\bar{\phi}_{\boldsymbol{F}}}\right)_{\mathbf{x} C 0}$ & $f_{\mathrm{RC}}$ & $f_{\text {moo }}$ \\
\hline 1.8 & 1.595902 & 1.325893 & 0.999861 & 0.999832 \\
\hline 1.6 & 1.484790 & $1 \cdot 303616$ & 0.999800 & 0.999814 \\
\hline 1.4 & 1.401965 & 1.272824 & 0.999714 & 0.999792 \\
\hline $1 \cdot 2$ & 1.328663 & $1 \cdot 238422$ & 0.999588 & 0.999764 \\
\hline 1.0 & 1.259559 & 1.199773 & 0.999388 & 0.999726 \\
\hline 0.8 & $1 \cdot 193202$ & $1 \cdot 157289$ & 0.999033 & 0.999669 \\
\hline 0.6 & $1 \cdot 130091$ & $1 \cdot 111750$ & 0.998294 & 0.999576 \\
\hline 0.4 & 1.072665 & 1.065726 & 0.996243 & 0.999391 \\
\hline
\end{tabular}

$\mathrm{RC}=$ Rectangular cell.

$\mathrm{ECC}=$ Equivalent cylindrical cell.

\section{DISCUSSION}

Because the thermal utilization of the lattices considered here are so close to unity, it is perhaps more meaningful to consider the disadvantage factor, $\bar{\phi}_{M} / \bar{\phi}_{T}$, in analysing the method. From Tables 1 and 2, we see that truncation at $k_{\max }=l_{\max }=3$ gives quite good results, which is encouraging, since if a very high order approximation were required the method would rapidly become overly cumbersome.

From Tables 3 and 4 we obtain a feeling for the validity of the equivalent cylindrical cell approximation, or rather, for the fuel-to-moderator ratio necessary before such an approximation becomes valid. It is perhaps better that these results speak for themselves, since each individual carrying out reactor calculations must decide for himself what accuracy is required. However, as the fuel-to-moderator ratio decreases, the ECC approximation improves, which is certainly to be expected on physical grounds.

The same method has been applied to problems in three-dimensional lattices (uranium spheres imbedded in moderator) and also to the problem of resonance interference. The details are given by Mannan.

Finally, it should be pointed out that recently similar methods have been proposed both for neutron problems (ADIR, 1966) and problems in heat transfer (AXFORD, private communication).

\section{REFERENCES}

ADIR J. (1966) Abstract, A. N. S. Student Conference, University of Illinois.

Amouyal A., Benoist P. and Hurowttz J. (1957) J. nucl. Energy 6, 79.

AXFORD R. Private communication.

Bors B. B. DU (1957) Report CEA 740.

Clark A. C. and Newmarch D. A. (1955) Report AERE-RP/R 1657.

GaLANIN A. D. (1956) Proceedings of the First International Conference on the Peaceful Uses of Atomic Energy, Geneva, P/666, Vol. 5, p. 477. United Nations, N.Y.

Glasstone S. and EdLund M. C. (1952) The Elements of Nuclear Reactor Theory, Prentice-Hall, New York.

MANNAN M. A. (1964) Thermal Utilization and Resonance Integrals in Nuclear Reactors, Sc.D. thesis, The University of Michigan.

Morse P. M. and Feshbach H. (1953) Methods of Theoretical Physics, MeGraw-Hill, New York.

Neumann H. (1957) Report HW-52048.

PaZY A. and Goshen S. (1961) Nucl. Sci. Engng 11, 357.

WEINBERG A. M. (1942) Report CP-330.

WIGNER E. P. (1933) Phys. Rev. 43, 804. 